Control-theoretic immune tradeoffs explain SARS-CoV-2 virulence and

$5 \quad{ }^{1}$ Computation and Neural Systems, California Institute of Technology; Pasadena, CA 91105.

$6 \quad{ }^{2}$ Division of Pulmonology, Critical Care, Allergy, and Sleep Medicine, Department of Medicine,

7 University of California-San Francisco; San Francisco, CA 94143.

$8{ }^{3}$ Department of Immuno-Oncology, City of Hope Comprehensive Cancer Center; Duarte, CA

991010.

$10{ }^{4}$ Control and Dynamical Systems, California Institute of Technology; Pasadena, CA 91105.

$11 *$ Corresponding author. Email: aasarma@caltech.edu 
13 Abstract: Dramatic variation in SARS-CoV-2 virulence and transmission between hosts has

14 driven the COVID-19 pandemic. The complexity and dynamics of the immune response present

15 a challenge to understanding variation in SARS-CoV-2 infections. To address this challenge, we

16 apply control theory, a framework used to study complex feedback systems, to establish rigorous

17 mathematical bounds on immune responses. Two mechanisms of SARS-CoV-2 biology are

18 sufficient to create extreme variation between hosts: (1) a sparsely expressed host receptor and

19 (2) potent, but not unique, suppression of interferon. The resulting model unifies disparate and

20 unexplained features of the SARS-CoV-2 pandemic, predicts features of future viruses that

21 threaten to cause pandemics, and identifies potential interventions.

Main Text:

Variations in virulence and transmission, shorthanded as the dual puzzles of

25 asymptomatic cases and superspreaders, have made SARS-CoV-2 infection and spread difficult

26 to predict and control (1-4). The relationship between pathogen virulence and transmission has

27 been a subject of longstanding speculation and formal study (5-7), and continues to be debated

28 in the context of variation in SARS-CoV-2 infection $(3,8-10)$. The complexity of the immune

29 response has impeded a unified mechanistic understanding of virulence, transmission, and

30 variation, relevant to SARS-CoV-2 and future emerging viruses (Figure 1). Here, we extend

31 techniques from control theory, a mathematical framework that has been used to analyze

32 complex feedback systems in both engineered and biological settings (11-14), to immune

33 biology to analyze SARS-CoV-2 virulence and transmission. 
We use control theory to uncover mechanisms that lead to variation in virulence and

38 transmission. Informally, we compute the best-case immune response, consolidating unmodeled

39 immune dynamics into a control function $K$ (Figure 2A). The best-case immune response

40 minimizes virulence and implicitly suppresses transmission. We implement mechanistic details

41 as constraints on the set of realizable control functions, and in this way identify mechanisms

42 (constraints) for which even a best-case $K$ yields virulence and transmission variation. This best-

43 case $K$ bounds any immune system model that we could have used, allowing us to pose rigorous

44 questions without a detailed model of immune dynamics. Formally, we consider the robust

45 control problem:

$$
v[t+1]=A_{\Delta}[t] v[t]+B_{\Delta}[t] u[t]+\delta[t]
$$

$$
u[t]=K(v[1: t])
$$

$v$ is a vector of viral loads, $u$ is the immune action, and new virus enters the system as $\delta$.

$49 A_{\Delta}$ and $B_{\Delta}$ are sets of time-varying matrices describing uncertain linearized dynamics. We

50 leverage theorems guaranteeing that the best-case $K$ always corresponds to a convex set, so that

51 the best-case $K$ computed from the set will be the best $K$ over all realizable functions (15).

The open-loop problem

We first consider the open-loop dynamics of viral replication, or equivalently $K=0$. We

55 model viral infection in the individual host as a three-step process: cell entry, replication in the

56 cell, and release of virus from the cell after an eclipse period $T_{e}(16,17)$. 


$$
\begin{gathered}
V \stackrel{k}{\rightarrow} \oslash \\
C_{I} \stackrel{k_{c}}{\rightarrow} \oslash \\
C_{S} \stackrel{k_{c}}{\rightarrow} \oslash
\end{gathered}
$$

63 single infected cell, where $(1-1 / \varphi)$ is the fraction of infected cells that constitutively turn over in

64 a single eclipse period.

$$
\begin{gathered}
A=\left[\begin{array}{cc}
1-k & k \alpha \\
I_{n-1} & 0
\end{array}\right] \\
\alpha=\frac{r \beta}{k \phi}\left[C_{s}\right]
\end{gathered}
$$

$\alpha$ scales linearly with $\left[C_{s}\right]$. A small fraction of respiratory epithelial cells are susceptible to SARS-CoV-2 when compared to rhinovirus, respiratory syncytial virus, and influenza (18-

69 22). A small susceptible cell fraction enables large relative variation consistent with reported

70 single-cell data $(20,21)$ (Figure 2B-C).

\section{The closed-loop problem}

We next consider the effects of immune control with innate extracellular effectors.

74 Higher $\alpha$ requires stronger immune responses to achieve a comparable effect on viral load

75 (Figure 2D). The immune response creates symptoms, which enable behavioral measures to

76 avoid infection (23). We use a highly simplified model of avoidance and isolation, emphasizing

77 the consequences of biological variation. We define transmission $R_{C L}$, where $w(t)$ is a warning

78 signal and $\gamma(t)=\exp (-p w(t))$. Initially, we take $w(t)$ to be a scaled norm of the immune response,

79 so that symptoms promote avoidance and isolation. 


$$
R_{C L}=\alpha_{r} \omega \int_{0}^{T} v(t) \gamma(t) d t
$$

We extend this simple behavioral model to address a virus with a long presymptomatic

82 period followed by uniformly severe infection (Figure 3A-B). Advance warning and isolation

83 measures can contain such a virus. However, fully asymptomatic cases make advance warning

84 more difficult. Fully asymptomatic cases need not be as contagious as presymptomatic-severe

85 cases to have this effect, and low rates of fully asymptomatic cases can be tolerated (Figure 3C). Interferon-based control varies less with $\alpha$ than extracellular responses, but interferon-

87 suppressed control varies more. Early interventions with exogenous interferon can potentially

88 reduce the eventual symptom burden in what would otherwise be severe cases (Figure 3D-E) . Taking these control layers together, we consider virulence and transmission as $\alpha$ varies.

90 Presymptomatic-severe high- $\alpha$ cases take a dominant role in spreading the pathogen, especially

91 where they interact with other high- $\alpha$ individuals (Figure 4).

\section{Discussion}

94 Other viruses

HCoV-NL63 and SARS-CoV-1 also bind to ACE2. HCoV-NL63 infection is

96 asymptomatic or cold-like (24), while SARS-CoV-1 infection is typically severe, with some

97 reported asymptomatic cases $(4,25)$. Viral infection in these cases could be biologically variable

98 with median effects that are too mild or too severe to be evident in clinical outcomes. SARS-

99 CoV-1 exhibits variable transmission, consistent with this interpretation (26). HCoV-NL63's

100 reduced virulence may result from a spike glycoprotein structure that decreases $\alpha(27)$.

101

102 Interferon signaling 
104 administration can reduce coronavirus infection severity (28-32). Our results suggest that

105 presymptomatic interferon could be particularly beneficial in averting severe outcomes.

106 Conversely, our results suggest a mechanism for harm from early immunosuppression in patients

107 with SARS-CoV-2, consistent with clinical trial evidence (33). Because of the ubiquity of

108 interferon suppression strategies in respiratory viruses, studies of control-guided interventions

109 could facilitate responses to future emerging viruses. 
112 1. C. Huang, Y. Wang, X. Li, L. Ren, J. Zhao, Y. Hu, L. Zhang, G. Fan, J. Xu, X. Gu, et al, 113 Clinical features of patients infected with 2019 novel coronavirus in Wuhan, China. The Lancet. 114 395, 497-506 (2020).

$115 \quad 2 . \quad$ W. Guan, Z. Ni, Y. Hu, W. Liang, C. Ou, J. He, L. Liu, H. Shan, C. Lei, D. S. C. Hui, et 116 al, Clinical Characteristics of Coronavirus Disease 2019 in China. New England Journal of 117 Medicine (2020), doi:10.1056/nejmoa2002032.

$118 \quad 3 . \quad$ W. J. Wiersinga, A. Rhodes, A. C. Cheng, S. J. Peacock, H. C. Prescott,

119 Pathophysiology, Transmission, Diagnosis, and Treatment of Coronavirus Disease 2019

120 (COVID-19). JAMA. 324, 782 (2020).

121 4. E. Petersen, M. Koopmans, U. Go, D. H. Hamer, N. Petrosillo, F. Castelli, M. Storgaard,

122 S. Al Khalili, L. Simonsen, Comparing SARS-CoV-2 with SARS-CoV and influenza pandemics.

123 The Lancet Infectious Diseases. 20, e238-e244 (2020).

124 5. R. M. Anderson, R. M. May, Coevolution of hosts and parasites. Parasitology. 85, 411$125426(1982)$.

126 6. S. Alizon, A. Hurford, N. Mideo, M. Van Baalen, Virulence evolution and the trade-off 127 hypothesis: history, current state of affairs and the future. Journal of Evolutionary Biology. 22, $128 \quad 245-259(2008)$.

$1297 . \quad$ J. J. Bull, A. S. Lauring, Theory and Empiricism in Virulence Evolution. PLoS

130 Pathogens. 10, e1004387 (2014).

1318 8. M. A. Johansson, T. M. Quandelacy, S. Kada, P. V. Prasad, M. Steele, J. T. Brooks, R. B. 132 Slayton, M. Biggerstaff, J. C. Butler, SARS-CoV-2 Transmission From People Without COVID13319 Symptoms. JAMA Network Open. 4, e2035057 (2021).

134 9. O. Byambasuren, M. Cardona, K. Bell, J. Clark, M.-L. McLaws, P. Glasziou, Estimating 135 the extent of asymptomatic COVID-19 and its potential for community transmission: Systematic 136 review and meta-analysis. Official Journal of the Association of Medical Microbiology and 137 Infectious Disease Canada. 5, 223-234 (2020).

138 10. A. L. Rasmussen, S. V. Popescu, SARS-CoV-2 transmission without symptoms. Science. 139 371, 1206-1207 (2021).

$140 \quad$ 11. H. El-Samad, H. Kurata, J. C. Doyle, C. A. Gross, M. Khammash, Surviving heat shock: 141 Control strategies for robustness and performance. Proceedings of the National Academy of 142 Sciences. 102, 2736-2741 (2005).

143 12. I. Lestas, G. Vinnicombe, J. Paulsson, Fundamental limits on the suppression of 144 molecular fluctuations. Nature. 467, 174-178 (2010).

145 13. F. A. Chandra, G. Buzi, J. C. Doyle, Glycolytic Oscillations and Limits on Robust 146 Efficiency. Science. 333, 187-192 (2011).

147 14. N. Li, J. Cruz, C. S. Chien, S. Sojoudi, B. Recht, D. Stone, M. Csete, D. Bahmiller, J. C. 148 Doyle, Robust efficiency and actuator saturation explain healthy heart rate control and 149 variability. Proceedings of the National Academy of Sciences. 111, E3476-E3485 (2014).

150 15. Y.-S. Wang, Matni N., J. C. Doyle, A System-Level Approach to Controller Synthesis.

151 IEEE Transactions on Automatic Control. 64, 4079-4093 (2019).

152 16. P. Baccam, C. Beauchemin, C. A. Macken, F. G. Hayden, A. S. Perelson, Kinetics of 153 Influenza A Virus Infection in Humans. Journal of Virology. 80, 7590-7599 (2006).

154 17. M. A. Nowak, S. Bonhoeffer, A. M. Hill, R. Boehme, H. C. Thomas, H. McDade, Viral 155 dynamics in hepatitis B virus infection. Proceedings of the National Academy of Sciences. 93, 156 4398-4402 (1996). 

D. Conley, Y. Mori, J. Seita, et al, A molecular cell atlas of the human lung from single-cell RNA sequencing. Nature. 587, 619-625 (2020). 351-360 (2008).

162 20. C. Muus, M. D. Luecken, G. Eraslan, L. Sikkema, A. Waghray, G. Heimberg, Y.

163 Kobayashi, E. D. Vaishnav, A. Subramanian, et al, Single-cell meta-analysis of SARS-CoV-2

164 entry genes across tissues and demographics. Nature Medicine. 27, 546-559 (2021).

165 21. J. C. Smith, E. L. Sausville, V. Girish, M. L. Yuan, A. Vasudevan, K. M. John, J. M. Sheltzer, Cigarette Smoke Exposure and Inflammatory Signaling Increase the Expression of the SARS-CoV-2 Receptor ACE2 in the Respiratory Tract. Developmental Cell. 53, 514-529.e3 (2020). 22. S. Kasela, V. E. Ortega, M. Martorella, S. Garudadri, J. Nguyen, E. Ampleford, A. Pasanen, S. Nerella, K. L. Buschur, I. Z. Barjaktarevic, et al, Genetic and non-genetic factors affecting the expression of COVID-19 relevant genes in the large airway epithelium (Cold Spring Harbor Laboratory, 2020; http://dx.doi.org/10.1101/2020.10.01.20202820). Immunology. 35, 457-464 (2014).

24. R. K. Dare, A. M. Fry, M. Chittaganpitch, P. Sawanpanyalert, S. J. Olsen, D. D. Erdman, Human Coronavirus Infections in Rural Thailand: A Comprehensive Study Using Real-Time Reverse-Transcription Polymerase Chain Reaction Assays. The Journal of Infectious Diseases. 196, 1321-1328 (2007). Asymptomatic SARS Coronavirus Infection among Healthcare Workers, Singapore. Emerging Infectious Diseases. 11, 1142-1145 (2005).

182 26. J. O. Lloyd-Smith, S. J. Schreiber, P. E. Kopp, W. M. Getz, Superspreading and the effect of individual variation on disease emergence. Nature. 438, 355-359 (2005). 27. A. B. Gussow, N. Auslander, G. Faure, Y. I. Wolf, F. Zhang, E. V. Koonin, Genomic determinants of pathogenicity in SARS-CoV-2 and other human coronaviruses. Proceedings of the National Academy of Sciences. 117, 15193-15199 (2020). Abbasian, H. Kazemzadeh, M. S. Yekaninejad, A Randomized Clinical Trial of the Efficacy and Safety of Interferon $\beta-1 \mathrm{a}$ in Treatment of Severe COVID-19. Antimicrobial Agents and Chemotherapy. 64 (2020), doi:10.1128/aac.01061-20. Gabbay, D. E. Davies, S. T. Holgate, L.-P. Ho, et al, Safety and efficacy of inhaled nebulised interferon beta-1a (SNG001) for treatment of SARS-CoV-2 infection: a randomised, doubleblind, placebo-controlled, phase 2 trial. The Lancet Respiratory Medicine. 9, 196-206 (2021). 30. I. F.-N. Hung, K.-C. Lung, E. Y.-K. Tso, R. Liu, T. W.-H. Chung, M.-Y. Chu, Y.-Y. Ng, J. Lo, J. Chan, A. R. Tam, et al, Triple combination of interferon beta-1b, lopinavir-ritonavir, and ribavirin in the treatment of patients admitted to hospital with COVID-19: an open-label, randomised, phase 2 trial. The Lancet (2020), doi:10.1016/s0140-6736(20)31042-4.

31. R. Channappanavar, A. R. Fehr, R. Vijay, M. Mack, J. Zhao, D. K. Meyerholz, S. Perlman, Dysregulated Type I Interferon and Inflammatory Monocyte-Macrophage Responses Cause Lethal Pneumonia in SARS-CoV-Infected Mice. Cell Host and Microbe. 19, 181-193 (2016). 
203 32. R. Channappanavar, A. R. Fehr, J. Zheng, C. Wohlford-Lenane, J. E. Abrahante, M.

204 Mack, R. Sompallae, Jr. McCray Paul B., D. K. Meyerholz, S. Perlman, IFN-I response timing

205 relative to virus replication determines MERS coronavirus infection outcomes. Journal of

206 Clinical Investigation. 129, 3625-3639 (2019).

207 33. Dexamethasone in Hospitalized Patients with Covid-19. New England Journal of

208 Medicine. 384, 693-704 (2021).

209 
A
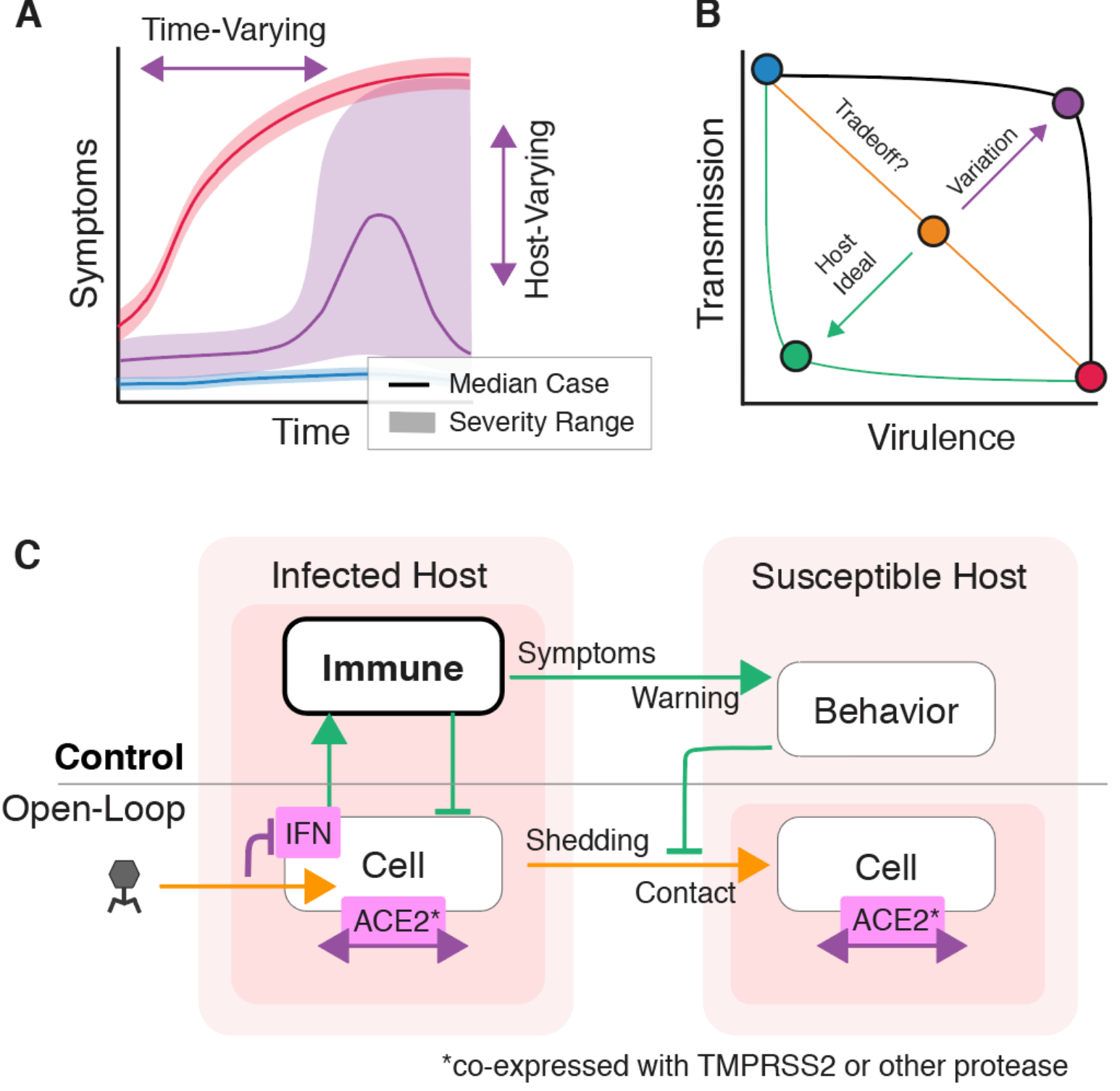

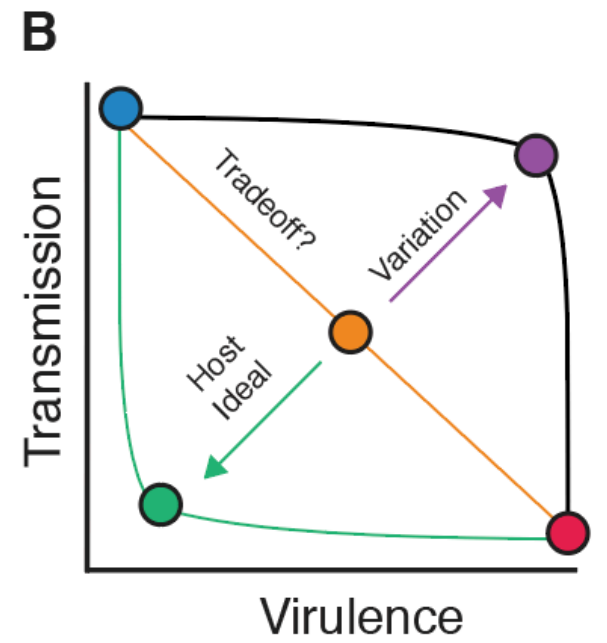

Virulence

213 Fig. 1. A control theory framework to analyze virulence and transmission.

214 (A) Schematic time-series representations of symptoms for three viruses. The red and blue cases

215 have relatively low variation across hosts and across time. The purple case, schematically like

216 SARS-CoV-2, varies across hosts and across time, suggesting variation in host immune

217 responses. 
218 (B) An apparent tradeoff between virulence and transmission can result from host immune

219 responses. However, this tradeoff depends on host control mechanisms, and can be made more

220 favorable to the host population or more favorable to the virus.

221 (C) A block diagram shows the relationships between virulence and transmission control in two

222 hosts, one infected with SARS-CoV-2 and one susceptible. The dynamics without control are

223 shown in the lower half of the diagram, and the control responses in the upper half. Immune

224 responses suppress shedding, create symptoms, and allow behavioral responses. 


\section{A}
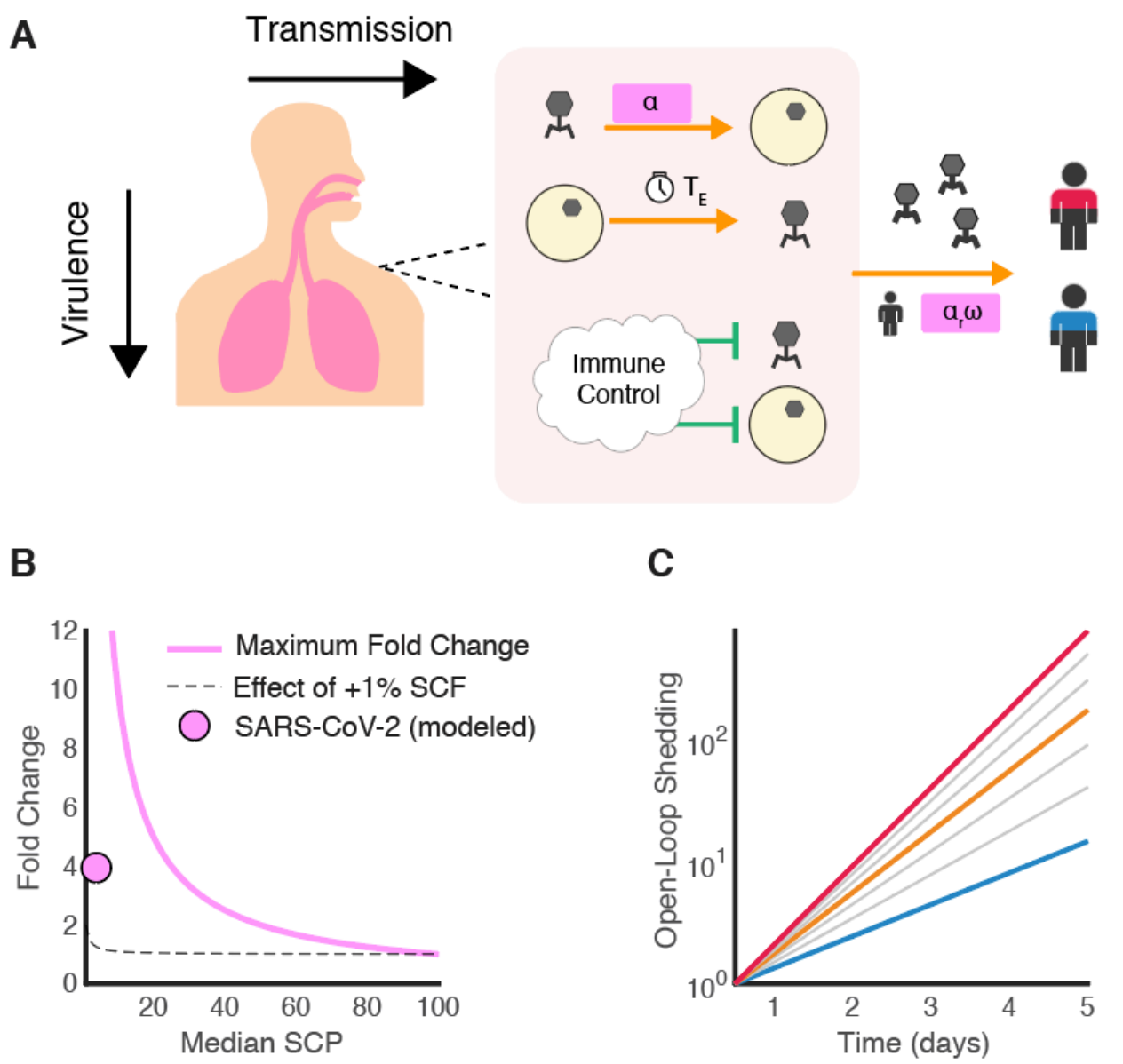

D
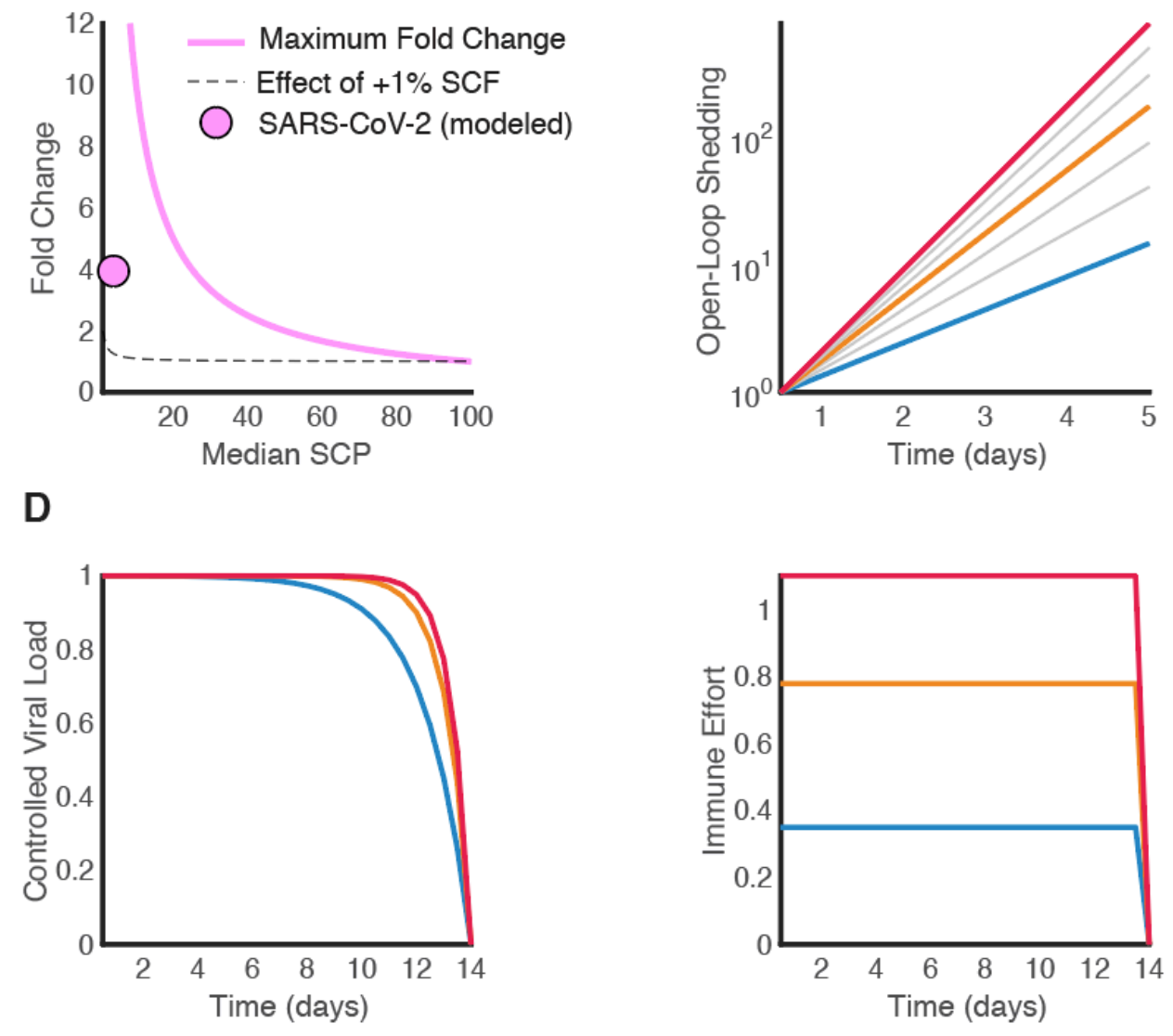
232 (A) Within each host, well-characterized kinetics govern viral replication. Viruses enter cells,

233 replicate, exit after a delay, and degrade in the extracellular space. These kinetics are coupled to

234 immune responses, for which we compute best-case bounds with control theory.

235 (B) A low susceptible cell percentage (SCP) in the host enables variation. The maximum fold-

236 change deviation from the median and the effect of a small fluctuation both grow as the median

237 SCP approaches $0 \%$.

238 (C) An open-loop model removes all control elements and considers the underlying dynamics.

239 Open-loop variation in viral shedding varies dramatically on relevant time-scales, amplifying

240 variations in SCP.

241 (D) Ideal extracellular immune control can create similar, low-variation viral load trajectories

242 between hosts, but these similar trajectories require differing immune effort. The underlying

243 open-loop dynamics directly shape virulence. 
A

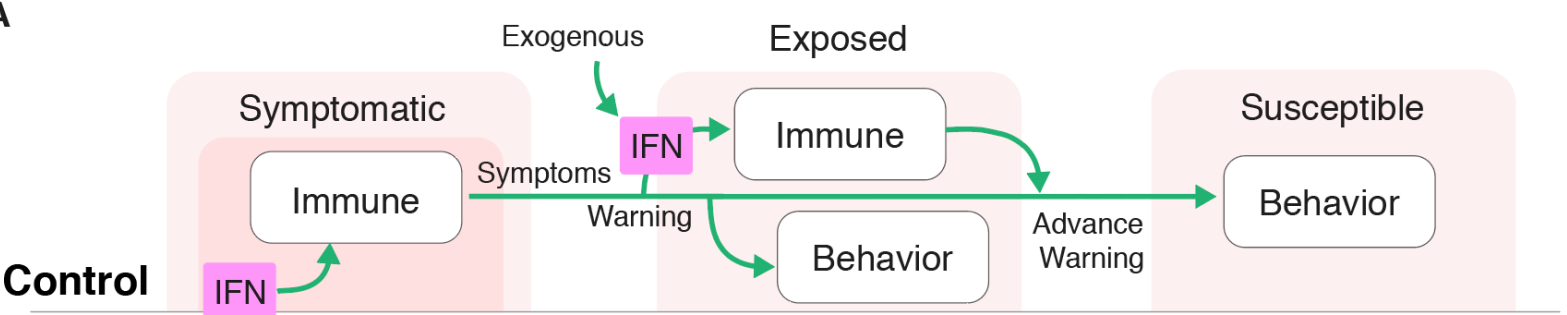

B

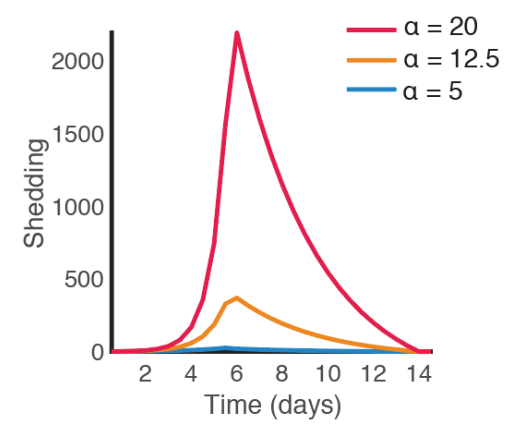

C
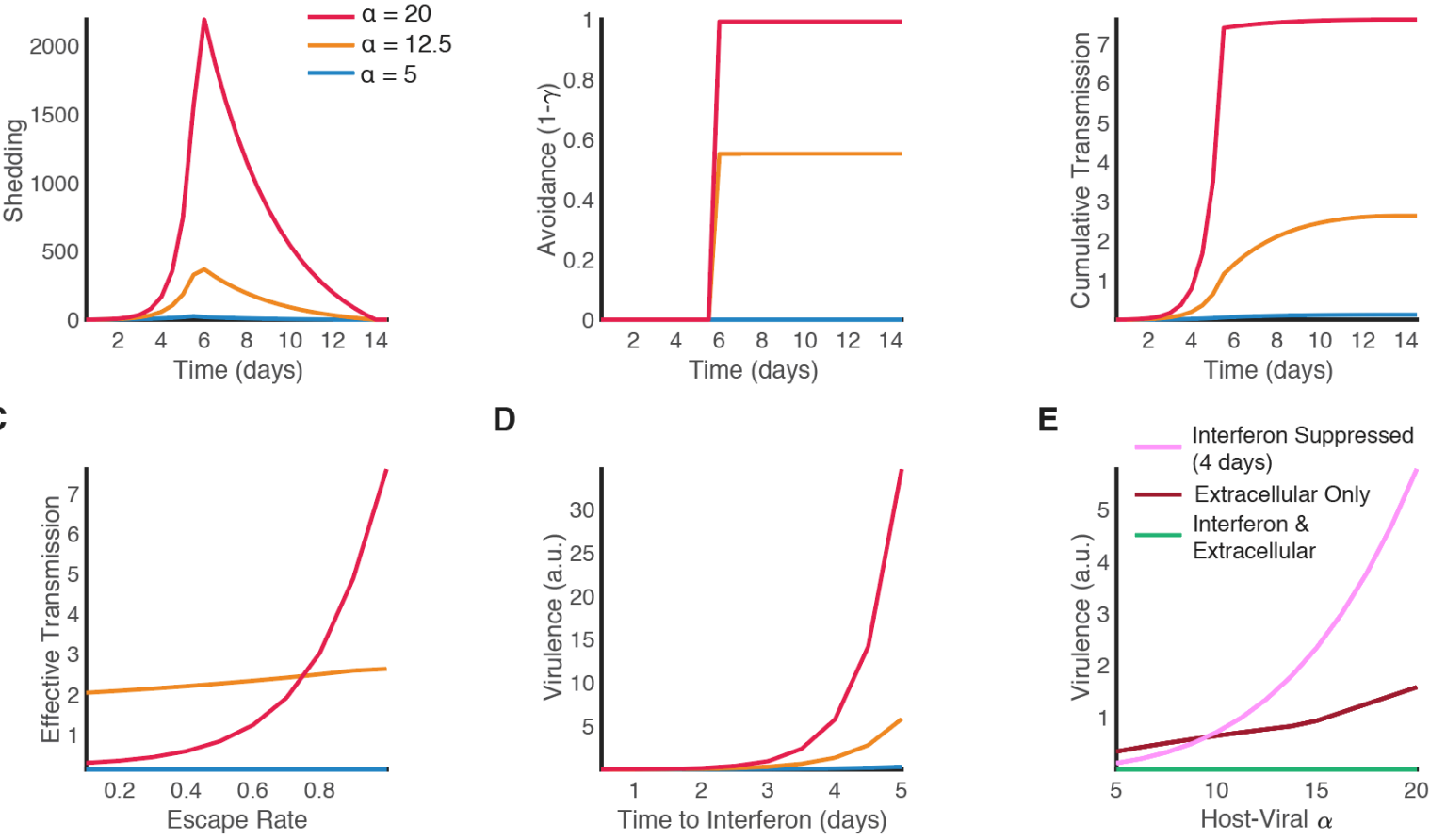

D

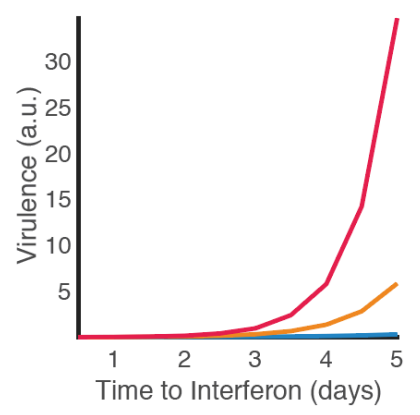

E

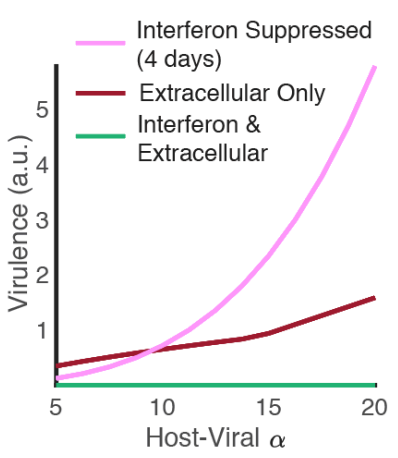

245 Fig. 3. Layered control of virulence, transmission, and variation.

246 (A) When all infected hosts are eventually symptomatic, advance warning allows isolation and

247 potentially treatment measures in pre-symptomatic individuals.

248 (B) Interferon-suppressed responses allow an extended period of viral replication and shedding

249 during which avoidance behaviors are not possible (without advance warning). Transmission can

250 be computed from the viral and immune trajectories.

251 (C) Advance warning can reduce the effective transmission rate of presymptomatic individuals,

252 but asymptomatic cases facilitate escape. As the rate of escape increases, the effective

253 transmission from presymptomatic-severe individuals increases sharply. 
254 (D) Timing is a crucial determinant of interferon efficacy. Presymptomatic exogenous interferon

255 administration can potentially reduce the eventual symptom burden in an individual who would

256 otherwise experience severe disease.

257 (E) Extracellular immune responses vary more with $\alpha$ than interferon-based immune responses,

258 but interferon-suppressed responses vary most. 


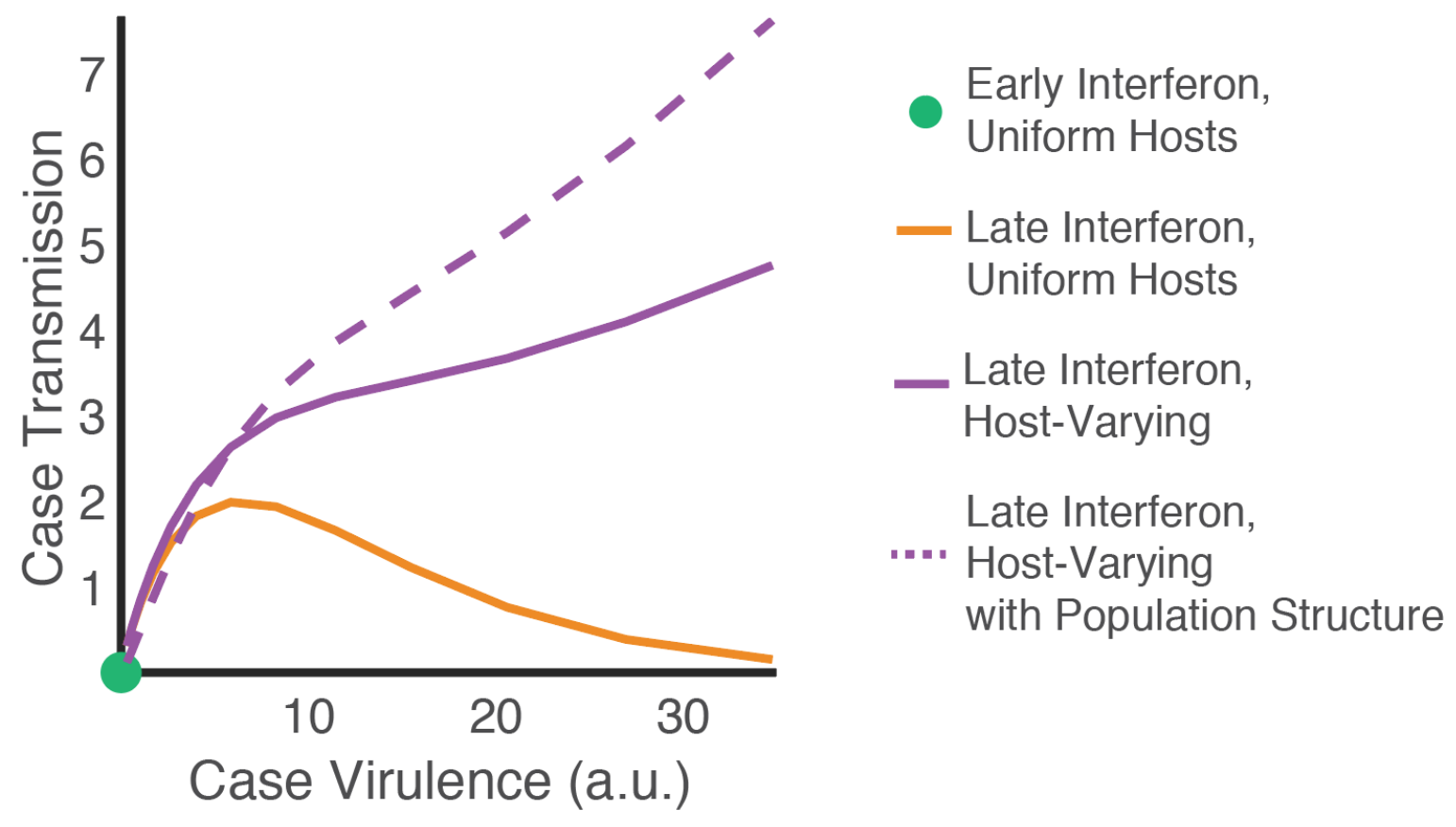

261 Fig. 4. Virulence and transmission depend on host control strategies.

263 The relationship between virulence and transmission depends on control conditions in individual

264 hosts. If immune control is ideal for the host, replication is quickly blocked by interferon and

265 neither serious symptoms nor substantial transmission occur. With interferon suppression,

266 transmission peaks at low virulence. With interferon suppression and host variation, however,

267 transmission is higher and peaks at higher virulence. This effect is amplified when high- $\alpha$

268 individuals interact, leading to both high presymptomatic shedding and high susceptibility. 